\title{
Cardiovascular specialist 2025. Basics of training stay the same, logistics improve
}

\author{
Adam Stys ${ }^{1}$, Tomasz Stys ${ }^{2}$ \\ ${ }^{1}$ Chief of Cardiology Division, Program Director of Cardiovascular Disease Fellowship, \\ Professor of Medicine, University of South Dakota Sanford School of Medicine, Sioux \\ Falls, USA \\ ${ }^{2}$ Cardiology Division, Director of Cardiovascular Services at Sanford University Medical Center, Professor of Medicine, \\ University of South Dakota Sanford School of Medicine, Sioux Falls, USA
}
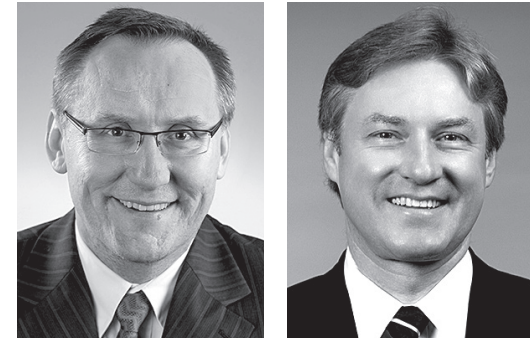

Kardiochirurgia i Torakochirurgia Polska 2016; 13 (2): 95-97

\section{Introduction}

Cardiovascular disease is the number one killer in developed countries. The need for well-trained specialists is obvious due to the societal impact of the disease. Training of cadres of future cardiovascular specialists is a daunting task. The ever-changing technological landscape and fast growing literature make it even more challenging. Time and effort invested into training such a specialist should pay off to society in improved quality of life and longevity. We would like to discuss the training of those who have just graduated from medical school and would be considered a "quality final product" about 2025.

\section{Candidate selection}

In our opinion, in order to be a good cardiologist, one has to develop a broad medical knowledge base first. Thus, prior sound internal medicine training is essential. As cardiovascular disease is a major subspecialty of internal medicine and its fellowship training is considered the most sought after in many countries, a good candidate base exists for the selection of future cardiologists. The importance of appropriate selection of an individual for training as a cardiovascular specialist cannot be underestimated. This is where success (or lack thereof) starts and can end.

The US National Resident Matching Program (NRMP, a private, non-profit organization established in 1952) and its derivative, the Medical Specialties Matching Program (MSMP), provide a platform for competitive distribution of spots for postgraduate training of physicians. It is arguably the largest, single, universal, countrywide, most comprehensive and fair system of obtaining specialty training positions. The NRMP uses a mathematical algorithm to place applicants into training positions. The algorithm is based on applicants submitting their preferences (known as the rank order lists). The programs submit their candidate pref- erence lists. Thorough examination of programs by candidates and vice versa, including candidate visits with formal interviews, occurs. No applicant could obtain a better outcome than the one produced by the algorithm. Research on the algorithm was the basis for awarding the 2012 Nobel Prize in Economic Sciences.

The match algorithm works in the best interests of the candidate, but also ensures that the coveted programs get quality candidates. It allows applicants and program directors to consider each other without pressure, creating an impartial venue for matching applicants' and program directors' preferences, and establishes a uniform date for appointments to programs, as well as ensuring that the applicants meet requirements for further training. Applications are uniform and electronic; the process is confidential and fosters fairness. The NRMP also provides policies and specialty-specific data for training programs and candidates. The 2015 Match was record breaking, with over 41,000 applicants competing for over 30,000 specialty training positions in 4,756 programs, including 50 subspecialties through its MSMP [1].

The more competitive a given specialty training is, the more applicants per spot; however, applicants can apply to many programs through the match. Programs can interview as many candidates as they deem appropriate. In our fellowship, each candidate is interviewed by all key clinical faculty members, spends time with current fellows, takes a tour of the facilities and is presented with the potential benefits/contract package by our program coordinator. The rank list is prepared by a committee that includes the fellowship faculty, Chair of the Department of Medicine, Internal Medicine Residency Director, hospital administration, program coordinator and Dean of Graduate Medical Education. Each candidate is openly discussed and then all key clinical faculty members assign him/her equivalent points that are then averaged out and a rank order list is created. 
Thus, the process is open and democratic, though the program director has the right to veto (so far not exercised). Our program receives about 200 applications per 2 spots in the first year of training. We choose about 25 to interview and rank on the match list about 15, usually matching 2 in the top 5 of our list.

\section{Training Quality Assurance}

The Accreditation Council for Graduate Medical Education (ACGME) is a private, non-profit organization that reviews and accredits graduate medical education (residency and fellowship) programs, and the institutions that sponsor them, in the United States. Established in 1981, the ACGME mission is to improve health care and population health by assessing and advancing the quality of resident physicians' education through accreditation. In academic year 20132014, there were approximately 9,600 ACGME-accredited residency and fellowship programs in 130 specialties and subspecialties at approximately 700 sponsoring institutions, with over 120,000 active residents and fellows.

Since 1981 the ACGME has been in charge of education quality of physicians, including future cardiologists in the USA, with their endeavors followed elsewhere in the world. Accreditation of a training program by the ACGME is necessary for its graduates to be eligible for board examination in their specialty. The ACGME has standardized the training requirements in detail and has been upgrading them regularly. Also it has been expanding into creating specific programs for post-cardiovascular disease training (i.e. interventional cardiology, electrophysiology). At present there are 193 accredited cardiovascular disease fellowships in the USA and 144 interventional cardiology fellowships, translating into about 3,000 trainees ( $2681+312$ positions).

The ACGME regularly upgrades its requirements for training program accreditation, working with specialty societies. From the cardiovascular disease standpoint, the American College of Cardiology (ACC) provides updated guidelines for specialty training, which usually are similar to or more stringent than ACGME ones. Then specific institutions/clinics/hospitals that employ newly graduated and certified specialists have their own credentialing requirements, which are usually in line with the ACGME and ACC, but can be more demanding, too. Thus, training of a specialist needs to be conducted with a long-term plan in mind, so that the individual is not only board exam eligible, but also can be given privileges to practice in his/her specialty anywhere.

ACGME training general competencies are the same for all specialties. The six pillars of training are patient care, medical knowledge, practice-based learning and improvement, interpersonal and communication skills, professionalism, and system-based practice. General requirements are followed by cardiology-specific, structured education, mandated, standardized, and monitored by the ACGME in every accredited program. The number and sort of conferences, length and content of rotations, and the training institution clinical learning environment are specified by the ACGME. The monitoring has become mostly electronic, with ACGME site visits regular but less frequent recently. The institutions that conduct training are also monitored and guided by the ACGME in order to provide an optimal learning environment for trainees. The ACGME has been active in a Sponsoring Institution 2025 project, which will further improve the quality of ACGME-accredited institutional sponsors of graduate medical education programs [2].

\section{Specifics of training in cardiovascular disease}

The modern cardiovascular disease specialist, whether going into the interventional field, electrophysiology or imaging practice, needs to be exposed to a large number of patients, studies, and procedures. The "numbers" experienced during training do translate, in our opinion, into quality of the specialist education. These are simple, though crude measures of the specialty training program quality as well as its graduates. The ACGME and ACC have specific requirements for these numbers; however, the rule of the more the better applies [3]. In our opinion, the usual numbers required by these organizations can be comfortably doubled in a cardiology fellowship. Electronic recording of the "volume" in training is optimal. This allows for easier tracking of for example procedures performed by a fellow and allows for quick corrections if need be, by the program director of a given fellow's training course.

Required rotations need to be intense, elective ones less so. Dedicated research time is a must. Publishing and presenting abstracts at national and international cardiovascular meetings should be required of the fellows. In our program every fellow needs to attend and present at ACC Annual Sessions and the American Heart Association Annual Meeting. They are required to publish a minimum of 5 manuscripts in peer-reviewed journals during their 3-year training.

Globalization of cardiovascular medicine is reflected in the organizations from different parts of the world writing together our practice guidelines. Modern training ideally should involve exposure to different institutions and healthcare systems, even abroad, in order to give an appropriate perspective and open the horizons. Visiting lecturers and trainees from other institutions enrich the training on site. Especially in the procedural-oriented specialty with rapid technology development that is cardiovascular disease, trainees' exposure to different institutions is very beneficial. As new technology and techniques evolve, one can always learn something different at a different place. I cannot forget a random statement made by a relatively young interventionist who thought that he has "nothing new to learn by going elsewhere". One cannot be more wrong. Every trip, every conference, every patient is a learning opportunity. Especially in early stages of a cardiovascular specialist career it is important to travel and mingle with peers as well as masters in the specialty. This experience stays for life and connections made then could benefit the trainee long term.

Evaluations of a fellow need to be obtained not only from clinical faculty, but also should come from represen- 
tatives of mid-level providers, medicine residents, nursing, technicians, and medical students. Fellows should be part of the teaching service for students and residents, optimally holding academic appointments at the medical school associated with the training institution. Regular and objective evaluation of fellows' learning progress can be optimally evaluated by the annual ACC In-Training Exam that for a fee gives the relative rank of fellows against their peers across the country and points out areas needing improvement. The training program should be evaluated by fellows in order to provide feedback for improvement. Our fellows fill out ACGME-required annual surveys (anonymous), but also participate in our medical school internal program evaluation.

In our opinion, the cardiovascular specialist of 2025 should receive comprehensive training including all aspects of cardiology. The recent trends of narrowing the training in fellowships and focusing on one field (for example on echocardiography) do not serve our patients well. Thus, we promote exposure to every aspect of imaging and invasive procedures for our fellows, with an opportunity to obtain expertise that would give them credentials in most institutions. The trends to subspecialize early in the career are not necessarily providing quality specialists. Moreover, in cardiovascular disease procedures we have seen convergence of specialties with widening of the scope of practice of cardiovascular specialists [4].

The practice of evidence-based medicine has seen a shift from specific database searches (e.g. Medline) on a specific clinical question towards applications of guidelines and broader practice updates. Fellows need to be taught how to exploit comprehensive clinical decision support resources like UpToDate in addition to classical medical literature searches [5].

Our cardiovascular fellowship program started over 3 years ago and has been built on the above ideas. Our first graduates have already passed the cardiovascular disease board exam, and have received board certification in nuclear cardiology, cardiac CT, echocardiography and vascular medicine. They have published over 10 manuscripts each and presented multiple abstracts at international meetings. Perhaps the best proof that the above ideas work is the fact that our fellows are last ACC Annual Meeting national champions in Fellows-In-Training Jeopardy. Team South Dakota, coming seemingly "out of nowhere", could compete and win with the best, well-established programs.

\section{Conclusions}

Cardiovascular specialist training is a major enterprise. Only a structured, standardized, multiple-check system can produce a good specialist. Further training standardization, compliance with accreditation requirements, institutional involvement and compliance are the bases for a good training program. Trainee's heavy case load, regular evaluations and standardized medical knowledge testing are prerequisites for success. Widening horizons with away rotations and national/international conferences plus promoting fluency on guidelines will further advance our trainees.

\section{Disclosure}

Authors report no conflict of interest.

\section{References}

1. Available at: http://www.nrmp.org/match-process/match-algorithm

2. Available at: http://www.acgme.org/acgmeweb

3. ACC 2015 Core Cardiovascular Training Statement (COCATS 4). A Report of the ACC Competency Management Committee. Vol. 65, No. 17, 2015.

4. Stys A. Kardiologia interwencyjna w obliczu rozwoju interwencji obwodowych. Konieczność współpracy pomiędzy specjalistami różnych dziedzin. Polish Heart Journal. Kardiol Pol 2006; 64: 344-346.

5. Available at: http://www.uptodate.com/home 